G.V. Kulinchenko, A.M. Maslennikov, V.A. Baguta

\title{
RESEARCH OF DYNAMIC PARAMETERS OF THE ELECTRIC DRIVE ON THE BASIS OF ROLLING ROTOR MOTOR
}

\begin{abstract}
Purpose. Development and investigation of a dynamic model of electric drive on the base of the rolling rotor motor (RRM) which reflects the positioning of the actuator of the locking and regulating equipment in time. Methodology. Analytical description of electromagnetic and mechanical processes in the electric drive during the RRM shaft movement by using a system of differential equations. Numerical imitation modeling with the processes visualization in the Matlab environment of the RRM rotor displacement with mechanical load in time. Results. It is shown that the degree of influence of the value of the load inertia on the dynamics of the object obtained by the waveform changes the rotation angle of the rotor and motor speed in time. The degree of influence of the value of the electromagnetic time constant of the dynamics of the positioning of the actuator, and the nature of transients during acceleration and fixing position of the rotor with a predetermined moment of inertia for different values of inductance. The effect of the ratio of electromechanical and electromagnetic time constants of the nature of the transition processes accompanying jog mode angular displacement of the drive shaft on the base of RRM. Originality. The lack of technical means to ensure acceptable accuracy time measurement of angular displacement shaft of the actuator in jog mode offset by using a laser meter which gives the opportunity to assess the adequacy of the dynamic model of the RRM. Practical value. The results of investigations allow to create a tool for optimization of structural, technical and hardware and software solutions for the improvement and modernization of the projected electric locking and regulating equipment. The direction for improving the dynamics of the drive on the basis of RRM is indicated providing for an increase in its torque characteristics of the motor by reducing the influence of the parameters of transients. References 10 , figures 9.

Key words: rolling rotor motor, time constant, positioning control, dynamics.
\end{abstract}

Анализируются динамические параметры электропривода на базе двигателя с катящимся ротором. Точность позиционирования такого мехатронного модуля обеспечивается в результате исследования факторов, определяющцх электромеханическую и электромагнитную постоянные времени. Экспериментальная оценка времени переходного процесса при управлении позиционированием модуля позволяет исследовать влияние различных параметров электропривода на его динамику. Библ. 10, рис. 9.

Ключевые слова: двигатель с катящимся ротором, постоянная времени, контроля позиционирования, динамика.

Introduction. A certain segment of the electric drive of valves is a mechatronic module combines the control system and executive of electric single-turn actuator (ESA) which consists of an asynchronous motor and gearbox. Tasks of improvement technical and economic performance of these modules require the search for alternative actuators, such as rolling rotor motors (RRM). Besides being able to combine the functions of the motor and the gearbox, which significantly improves the performance characteristics of the module, an additional advantage of the RRM is to achieve a predetermined starting torque at a relatively low starting current.

The main trend in the development of the RRM is to increase the power of these motors as their energy performance indicators are improved with an increase in motor power.

From the perspective of improving the RRM parameters used in the valves a decisive role in research play opportunities torque, which prevents violation of synchronous rotation and slippage of the rotor relative to the stator.

Problem definition. Defining the research aspects of dynamic parameters of trees and shrubs, we note that the methods and modeling techniques are determined by the specifics of the simulation object. Specific to the RRM which operates in the valve drive is a universal indicator of the dynamic that characterizes the performance - its response $\Pi[1]$ :

$$
\Pi=M_{r}^{2} / J_{r},
$$

where $M_{r}$ is the RRM torque; $J_{r}$ is the RRM rotor moment of inertia.
Evaluation of the dynamic characteristics of the actuator can be made at the time of acceleration and the engine stops at a given position, given the resistance moment and the moment of inertia of the load on the shaft of the RRM.

Such a formulation of the problem is typical for the step [2], induction [3] and DC motors used in mechatronic systems [4].

Features of formation of RRM torque [1] are small angular displacement of the motor shaft at predetermined control actions, not allow the use of the mentioned approaches to assess the dynamic parameters of the electric drive on the basis of RRM in jog mode

The relevance of research results from opportunities to increase torque RRM to values that are implemented in the ESA with the gearbox. This definition of the relevance of the benefit is due to the operating parameters of RRM on the parameters of ESA. The process of an adequate comparison data on the dynamics of the start-stop mode is inhibited imperfection of methods of assessing the dynamic parameters of the drive, especially with the random nature of the positioning valves (valve position control). Results of valves operating modes simulations make it possible to obtain data that are necessary for improving motor control algorithms based on RRM, as well as optimizing its design.

Known mathematical models of RRM based on the equations describing the electromagnetic processes of electric machines [6] allow to improve the design of trees

(C) G.V. Kulinchenko, A.M. Maslennikov, V.A. Baguta 
and shrubs on the analysis of the angular displacement of the rotor at high and low speeds in stationary modes.

At investigations of the influence of stator coils switching modes RRM its torque shown that in conditions of changing load [7] is its dynamic characteristics determine the operational capabilities of the transmitter in various fields. By selecting the settings of switching modes for RRM given design, the desired speed at different points of resistance on the motor shaft.

More effective to assess the dynamic parameters RRM seems a simulation model [8] in which As the result of solution of the equation

$$
U(t)=\frac{\partial \Phi(i, v)}{\partial i} \frac{d i}{d t}+\frac{\partial \Phi(i, v)}{\partial v} \frac{d v}{d t}+R \cdot i,
$$

where $U(t)$ is the supply voltage; $i$ is the winding current; $\Phi$ is the magnetic flux of the stator winding; $R$ is the winding resistance; $v$ is the rotor rotation angle, we can obtain electromagnetic torque values $M_{e}$ depending on the rotation angle:

$$
M_{e}(i, v)=\frac{\partial \int_{0}^{i} \Phi(i, v) d i}{\partial v} .
$$

The position of the rotor which is determined by the angle of rotation $v$ is calculated by solving the equations of equilibrium of torque on the motor shaft, whose members include the moment of resistance of the rotor movement, the dynamic moment of resistance, depending on the moment of inertia of the rotor and the load. The resulting static electromagnetic torque of this model does not reflect the nature of the transients which accompany switching operation of the rotor movement and depend on the electromechanical parameters of electric drive.

The goal of the work is to develop and investigate the drive dynamic model based on RRM which reflects the positioning of the valve actuator mechanism in time.

In addition, the results of the work provided for the formation of the requirements for the hardware and software components of the electric drive control systems on the basis of the RRM which is a tool to optimize the parameters of valve.

DKR transition from synchronous to asynchronous mode depends on the ratio values of the frictional force and the component of force of unilateral magnetic attraction (FUMA) creating a movement of the rotor on the stator bore. In the transition of the RRM in asynchronous mode accompanied by a rotor slippage the task of retaining the executive valve mechanism is in position by the action of the moment of resistance on the drive shaft. In this case, the objective of research is to assess the feasibility of the required value of the electromagnetic torque RRM.

Influence of magnetic properties of materials of RRM magnetic system on its dynamics. Since RRM shaft movement occurs as a result of the magnetic field of the stator to the rotor, it is necessary to assess the effect on the electro-dynamic performance parameters RRM its mathematical model. This approach stems from the fact that the absence of mechanical load on the motor corresponds to the idle mode in which the slip does not occur.
Increase of the RRM torque as a necessary condition to improve its dynamic parameters, is focused on achieving the optimal proportions of electromagnetic parameters that characterize the operation modes of the RRM.

The motor torque depends on the angular position of the rotor relative to the stator field and the value corresponding to the angle of magnetic induction in the air gap of the RRM [9]:

$$
B_{\delta}(\varphi)=\frac{2 \cdot F_{\delta} \cdot \mu_{0}}{\delta \cdot \operatorname{ch}^{n}(\varphi)},
$$

where $F_{\delta}$ is the MMF of the air gap in radial direction; $\delta$ is the air gap; $\varphi$ is the angular coordinate determining radial value of the air gap length; ch is hyperbolic cosine; $n$ is the number depending on the motor design.

Analysis of changes in the values of magnetic induction in the air gap range by changing the angle of rotation of the rotor shows that the change in rotation of the rotor $B_{\delta}$ induction are $6 \%$. Accordingly, taking into account the non-linear nature of the FUMA depending on the angle of rotation of the rotor, it is possible to estimate the range of variation for the two extreme values of the angle of rotation.

Using the magnetization curve of the steel rotor (curve 3, Fig. 1) we can calculate the value of the magnetic induction in the air gap between the stator and the rotor, depending on the intensity of the magnetic field for the minimum $(0.001 \mathrm{~mm})$ and maximum $(0.08 \mathrm{~mm})$ air gap which in Fig. 1 are displayed by lines 1 and 2 .

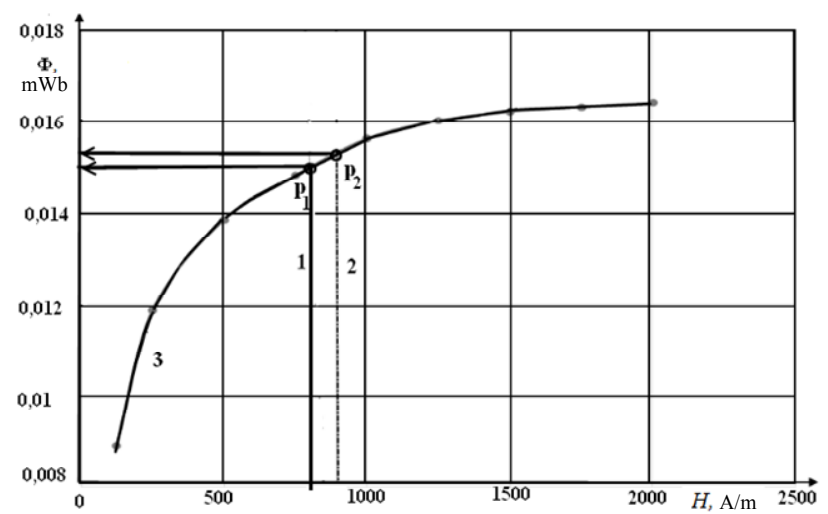

Fig. 1. Depending of the magnetic flux on the magnetic field strength

Two values of magnetic flux (points $\mathrm{p}_{1}$ and $\mathrm{p}_{2}$ ) corresponds to two values of magnetic flux $\Phi_{1}=0.015$ $\mathrm{mWb} ; \Phi_{2}=0.0155 \mathrm{mWb}$ and magnetic flux density $B_{1}=1.515 \mathrm{~T} ; B_{2}=1.565 \mathrm{~T}$.

This fact, with sufficient accuracy for practical purposes, enables approximate function flux density in the air gap of the stator and the rotor of the linear dependence of the angle of rotation of the rotor.

The value of the magnetomotive force $(\mathrm{MMF}) F_{\delta}$ of the stator winding, which causes the value of magnetic induction in the air gap depends on the value of the stator current $I$ flowing through the $N$ turns of the coils of the stator winding. Since the coil current when applying a voltage pulse is changed according to the law:

$$
I=\frac{U_{p}}{R_{N}}\left(1-e^{-t / \tau}\right),
$$


where $U_{P}$ is the voltage on the winding terminals with resistance $R_{N} ; \tau=L / R_{N}$ is the time constant; $L$ is the coil inductance. Under this law, the rate of rise of EMF in the air gap, a change in magnetic flux and magnetic induction will be determined by the time constant which depends on the parameters of the stator winding.

Thus, the desire to increase the torque by increasing the RRM MMF is limited not only design considerations but also increase the inductance coil which together with the winding resistance time constant, and hence the dynamics RRM.

In [9] it is shown that the transition process in the stator winding of much longer rotor rolling, so the influence of electro-mechanical time constant of the process dynamics can be neglected. However, increasing the mass of the rotor, and respectively, its moment of inertia $J_{r}$ which is essential for powerful engines disregard electromechanical time constant leads to errors in the estimates of the dynamic characteristics of the RRM.

The factors affecting the modes of RRM you should pay attention to the ratio of the electrical and electromechanical time constants, with time winding switching, since these parameters, together with the rotor friction regulate the mode change RRM from synchronous to asynchronous mode or slippage.

Formalization of the mathematical model of the RRM. Formulating objectives DKR simulations that determine the structure of the model, we take into account the fact that the investigated object is used in the composition of the gas throttling mechatronic module. This means that in addition to the requirement value of torque and holding torque pipeline throttle position, engine real part of a module must provide the necessary speed and positioning accuracy, that is, the proper control action pickup. Accordingly, model RRM should reflect the connection of said parameters from the control channels.

Specificity of functioning throttle module is the time change in the nature and direction of the mechanical stress. Therefore, developing a model should reflect the speed and mechanical characteristics in different modes of operation of the actuator based on the RRM.

Since the processes occurring in any motor, influence each other, for the study of dynamic modes mechatronic module is necessary to consider the relationship of energy balance equations and electromagnetic torque with the equations of motion of the components of the module. As a result of solving these equations numerically obtained time dependence, reflecting the dynamics of the process of functioning of the module with the RRM. Application of numerical modeling techniques is due to the nonlinear character of the equations describing the state of the trees and shrubs.

The structure of the model mechatronic module based RRM present scheme consisting of 4 blocks:

- block emulation movement of the magnetic field of the stator (BEFS);

- logic block (LB);

- emulation block of $B_{\delta}$ magnetic flux density in the air gap of the stator and rotor (BEMI);

- mechanical block (MB).
Mechanical block of the model, in accordance with traditional approaches to describing movement, can be represented by the following equations:

$$
\frac{d}{d t} \omega_{r}=\frac{1}{J_{m}}\left(T_{e}-T_{f}\right) ;(6) \quad \frac{d}{d t} \varphi=\omega_{r},
$$

where $\omega_{r}$ is the rotor rotation angular frequency; $J_{m}$ is the total reduced moment of inertia of the rotor and the load; $T_{e}, T_{f}$ are the electromagnetic torque and the moment of resistance to rotation; $\varphi$ is the rolling angle of the rotor relative to the stator field.

In the environment of MATLAB Simulink simulation corresponds to a block diagram shown in Fig. 2 corresponds to (7).

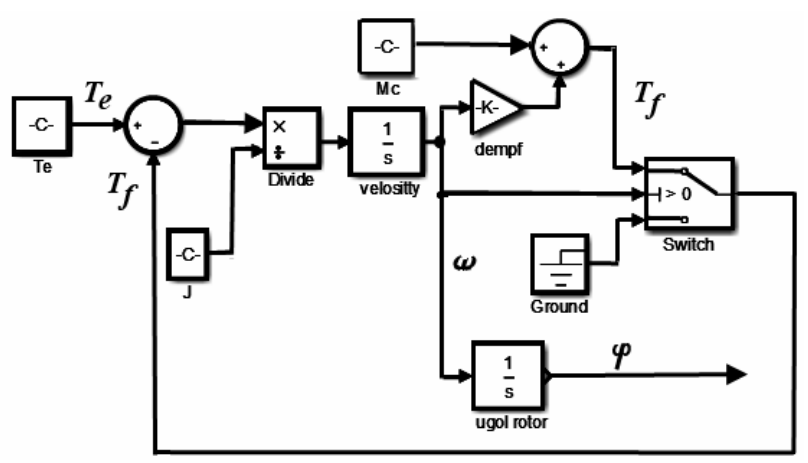

Fig. 2. Block diagram of the modeling MB

A feature of modeling MB RRM is a reflection of fixing the rotor position as a result of the voltage pulse off applied to the stator coil. At the time when the electromagnetic torque $T_{e}=0$ but continues to operate the time $T_{f}$ resistance, the rolling speed of the rotor begins to decline. After the rotor speed reaches zero, the circuit pattern using the element MB Switch deactivated the moment of resistance.

As can be seen from Fig. 2, in the membrane via the damping factor is taken into account the time dependence of the resistance on the rotor speed.

The block of emulation of magnetic flux density $B_{\delta}$ reflects the change in the value of the magnetic flux density in the air gap of the stator and rotor rolling. These changes are described by (4).

Work BEMI synchronized switching pulse generator motion control circuit RRM. As a result of applying a voltage pulse in the windings of the stator coils RRM, the current MMF and forming the magnetic induction in the air gap varies exponentially (5). Model changes can represent aperiodic link with a time constant $\tau$ winding. Actually, it is this constant corresponds to the electromagnetic time constant of the RRM.

For the time varying magnetic flux density values, take into consideration the magnitude of the air gap, which also changes periodically as a result of movement of the rotor. Calculations are performed in the gap $\delta$ by linear interpolation of $\delta$ depending on the load angle $\Theta$ which is connected to the rotor rotation angle $\varphi$. BEMI simulation diagram is shown in Fig. 3.

Emulation block of motion vector of the stator magnetic field displays discrete changes of the magnetic field vector angle in accordance with a frequency set by external switching pulse generator. 


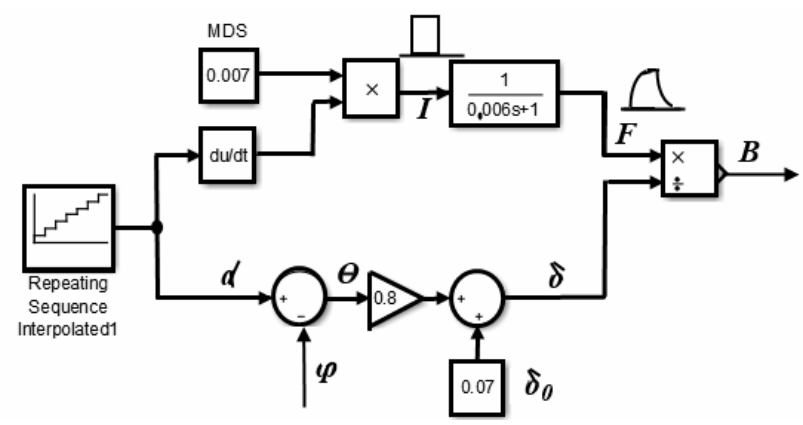

Fig. 3. BEMI diagram

In accordance with the switching rules 8 stator coil RRM angle displacement vector of the magnetic field of the stator is $\alpha=\pi / 4$. For the rotor in one direction or another switching pulse generator (Fig. 3, 4) generates signals which effect movement provides discrete induction vector at an angle $\alpha=\pi / 4$. Violation of balance of forces acting on the rotor caused by switching the coils of the stator winding sets it in motion, resulting in a change of the current position of the rotor angle $\varphi$ with the changes and load angle $\Theta=(\alpha-\varphi)$.

Electromagnetic RRM time dynamic model by moving the magnetic induction vector associated with the load angle $\Theta$ calculated by [1]:

$$
T_{e}(\Theta)=\frac{B_{\delta}^{2} \cdot S \cdot d_{r}}{2 \mu_{0}} \cdot \sin \Theta,
$$

where $S$ is the surface area through which the main magnetic flux; $d_{r}$ is the rotor diameter.

As a result, the time can create electromagnetic simulation RRM circuit shown in Fig. 4.

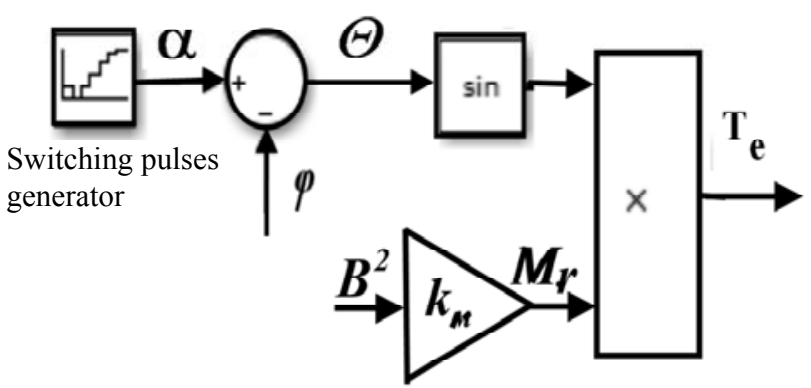

Fig. 4. Diagram of the RM electromagnetic torque modeling

Since the objective of the simulation is to study the dynamics of the parameters of the RRM, the results of modeling should reflect the interaction of acceleration and deceleration processes of the rotor over time. Characteristic of these processes is a change in velocity and acceleration values of the rotor not only in time but also in direction. The logic of the relationships of parameters and ranges of restrictions are implemented in the logic block $(L B)$. LB model allows reflect the direction of rotation of the rotor switch RRM and to exclude the value of the model parameters, which are contrary to the physical meaning of the engine operation. These problems are solved by a combination of logic functions simulation environment Matlab.

LB Interaction with other blocks of the model is reflected in the overall block diagram of the dynamic model RRM shown in Fig. 5.
The input parameters of the model are given by the characteristics of the RRM and the parameters of mechatronic module of valve. The simulation results are obtained in the form of time-varying output dynamic model parameters - the torque and rotational speed of the rotor angle RRM.

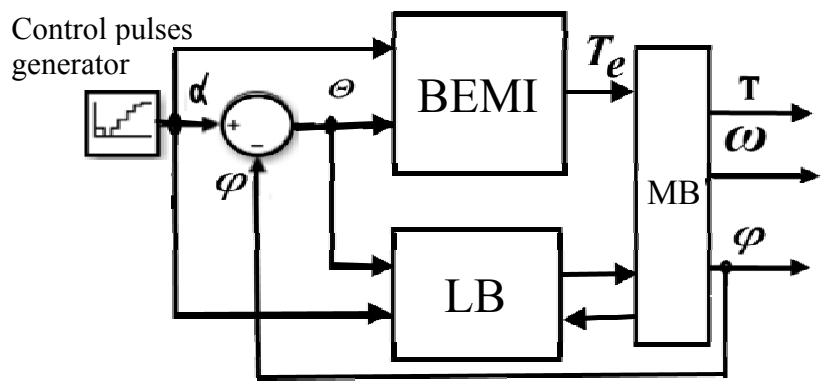

Fig. 5. Block diagram of the RRM dynamic model

These changes are the output parameters are visualized using Matlab user interface environment. The nature of the perturbation modeling object parameter is defined on the basis of the simulation tasks.

The simulation results. Exploring the value of the degree of influence of the dynamic moment of inertia of the object, received waveform changes the angle of rotation, trees and shrubs of the rotor speed with time for various values of the moment of inertia $J_{m}$ (Fig. 6).

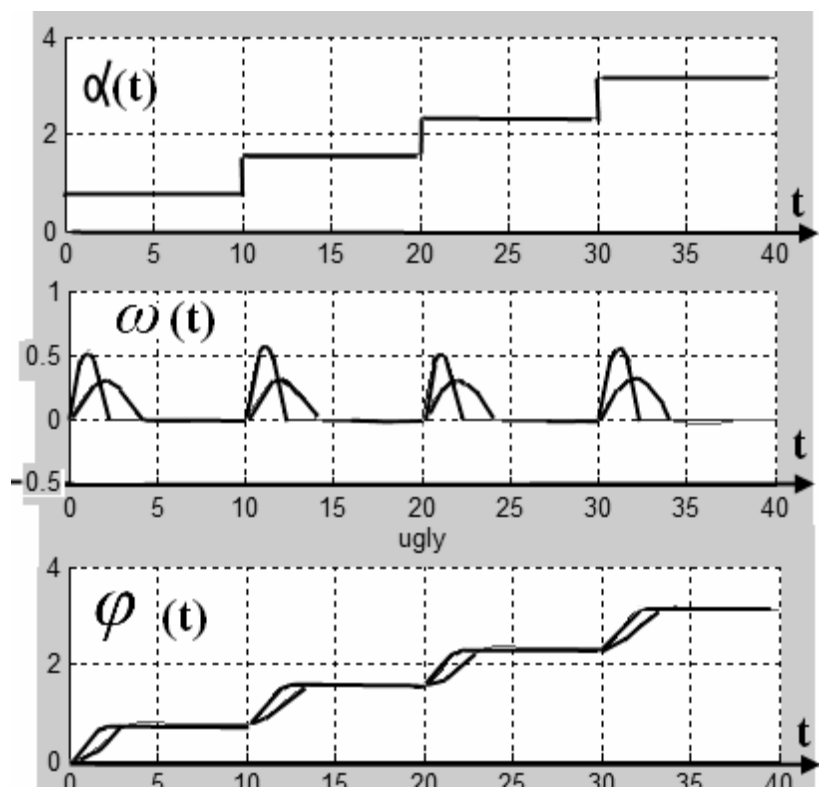

Fig. 6. Waveforms of changes the angular velocity and the angle of rotation of the rotor windings during commutation

Fig. 7,a shows the changes in the angle of rotation of the induction vector control, Fig. 7,b - electromagnetic torque $T_{e}$ fluctuations Fig. 7,c - the angular speed variation, Fig. 7, $d$ - the angle of rotation of the rotor for different values of the load torque. From these data it follows that increasing the time constant of the module due to the shaft torque load up to a certain value, it is necessary to limit the switching frequency of the windings. Fig. 7,c,d is evident that under certain loads on the shaft of the RRM control rotor motion is meaningless because there is no fixation of the rotor in the previous position. 
To assess the impact of the value of the electromagnetic time constant of the dynamics of the positioning of trees and shrubs, consider the nature of the transients during acceleration and fixing position of the rotor with a predetermined moment of inertia for different inductance values (Fig. 8).

In relative terms in Fig. 8, $a$ shows the variation of the electromagnetic moment in time; $b$ - the resultant moment, $c$ - change of angular velocity, $d$ - the angle of rotation of the rotor to the winding time constant $\tau=5 \mathrm{~ms}$ and $\tau=50 \mathrm{~ms}$.
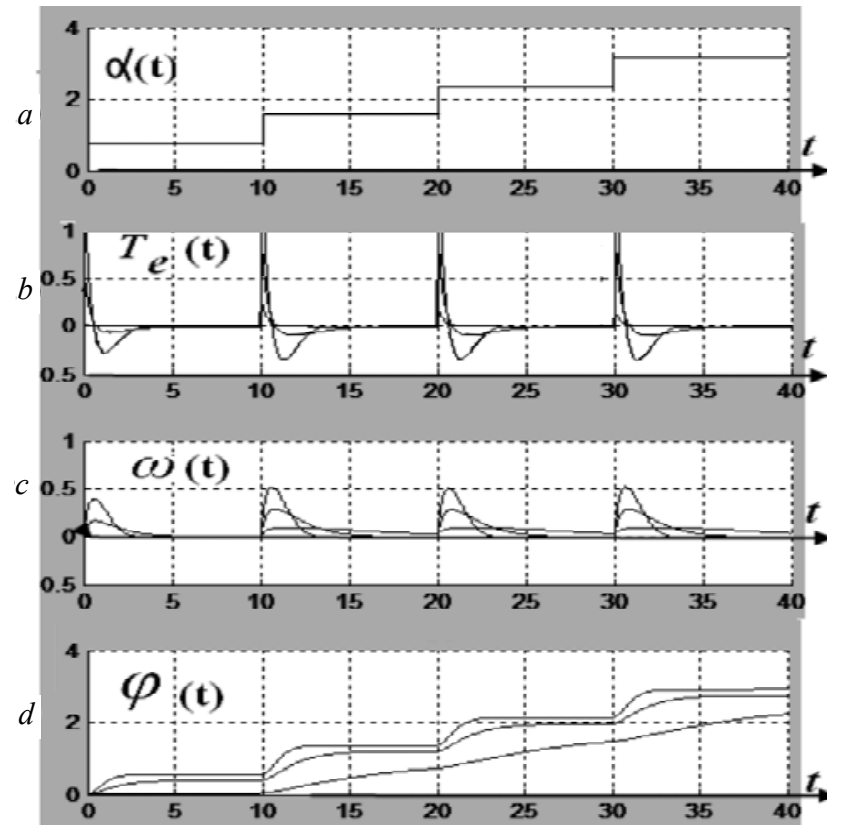

Fig. 7. Waveforms of changes of the torque, the rotor speed angle and rotation angle

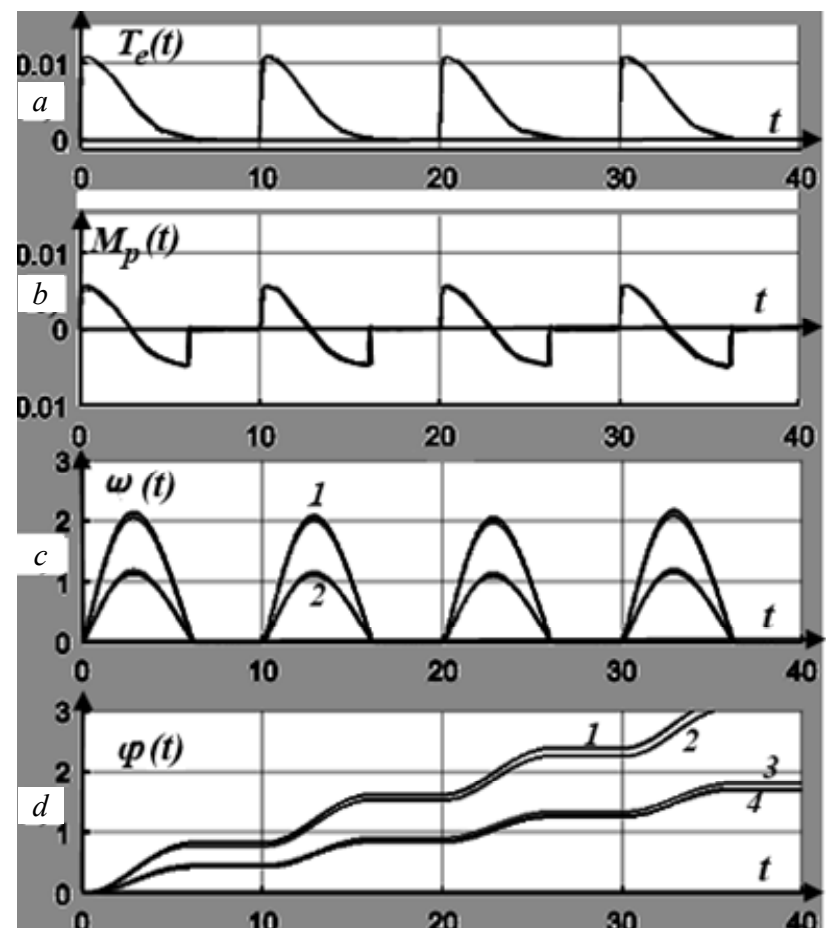

Fig 8. Waveforms of changes of electromagnetic torque, angular velocity and the angle of rotation of the rotor
The curves 1, 2 on the waveform $8, d$ correspond to a change in the angle of rotation for the inertial torque $J_{m 1}$, and curves 3 and 4 - for the moment of inertia $J_{m 2}$.

The values of angular velocity for a moment of inertia $J_{m 1}$ (curve 1) and $J_{m 2}$ (curve 2) in Fig. 8 differ appreciably, while, as a result of the difference signals changes inductance value on the order of ( $\tau=5 \mathrm{~ms}, \tau=50 \mathrm{~ms}$ ) unobtrusive.

It should be noted that an increase in inductance of RRM more $500 \mathrm{mH}$ (which is no practical necessity) also degrades engine dynamics, particularly in the starting torque / braking.

From the transient analysis implementations obtained shows that the positioning control device of valve in which RRM are used, should be developed taking into account the ratio of electromechanical and electromagnetic electromechanical time constants valve drive. Despite the fact that the value of the inductance of the stator winding in MMF electrical state significantly affects the formation of MDS stator winding RRM increasing the electromechanical constant as a result of increased mechanical load increases the transient driving, up to a loss of accuracy of positioning of the shaft.

To assess the adequacy of the dynamic model RRM used experimental setup diagram is shown in Fig. 9.

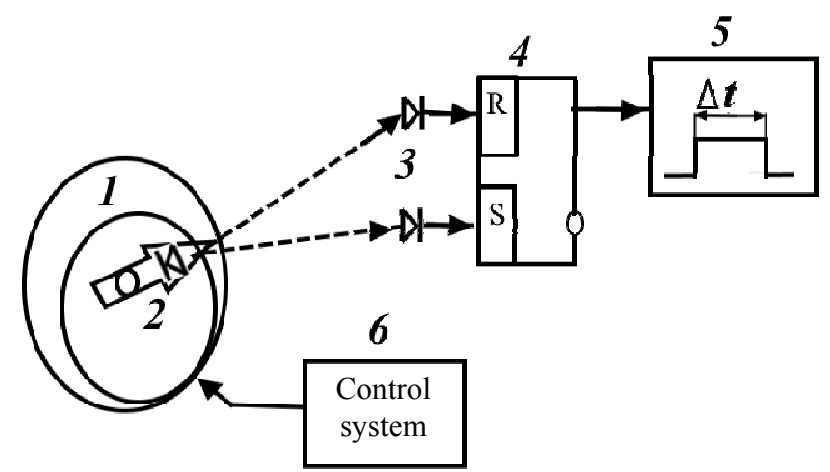

Fig. 9. Diagram of the change of duration of the RRM shaft displacement on the given angle

On the SSR shaft 1 laser emitter 2 is mounted the movement of the beam is perceived laser photodetectors 3. When moving the laser beam, the locking position of the shaft RRM through radiation photodetectors state is changed by trigger 4 . Use measuring time intervals 5 , which serves as a storage oscilloscope RIGOL SDS 1022DL, estimated travel time from one shaft RRM position to another.

This time clearly depends on the time of the transient change of the rotor position. The values of angular displacement are determined by the coefficient of reduction of the RRM, respectively, RRM design features. The need to use the laser measuring instrument is the difficulty of measuring small values of angular shaft displacements RRM feeding unit of the switching pulse.

With the help of the described installation (Fig. 9) to assess the impact of different windings switching modes RRM on the nature of transient electric [10] is carried out. The results of the time correction of the transition process, which takes into account the ratio of the electrical and electromechanical time constants, indicate the possibility 
of improving the dynamic parameters of the drive and increase its torque. Continued use of the results of modeling the dynamics of jog mode electric drive on the basis of the RRM opens the possibility of using energy-efficient control algorithms.

Conclusions. A dynamic model of the electric drive on the basis RRM reflecting the positioning of the drive shaft at the time taking into account the influence of the valve parameters on the parameters transients is developed.

Data obtained as a result of the simulation provide an opportunity to formulate the requirements for the hardware and software components of the electric drive control system based on the RRM.

Experimental evaluation of the time of transients in the management of valve positioning modules allow us to state that the improvement of the electric drive on the basis of the dynamics of RRM aimed at increasing its torque, it is advisable to carry out on the basis of hardware and software systems that provide reducing the influence of the characteristics of RRM on the parameters of transients.

\section{REFERENCES}

1. Nanij V.V., Miroshnichenko A.G., Juhimchuk V.D. Egorov A.V., Dunev A.A., Maslennikov A.M., Pototskiy D.V. Aspects of the design and testing of engines with a rolling rotor. Bulletin of NTU «KhPI». Series: Problems of electrical machines and apparatus perfection. The theory and practice, 2010, no.55, pp. 84-88. (Rus).

2. Sokolov A.V. Dynamic models of stepper motors. Bulletin of the South Ural State University, 2012, no.16, pp. 116-119. (Rus).

3. Palpankar P.M., Ghanmare R.U., Makade N. A generalized dynamic model of induction motor using Simulink. ITSI Transactions on Electrical and Electronics Engineering, 2013, Vol.1, Iss.5, pp. 118-122.

4. Farhan A. Salem. Dynamic modeling, simulation and control of electric machines for mechatronics applications. International journal of control, automation and systems, 2013, Vol.1, no.2, pp. 30-42.

How to cite this article:

Kulinchenko G.V., Maslennikov A.M., Baguta V.A. Research of dynamic parameters of the electric drive on the basis of rolling rotor motor. Electrical engineering \& electromechanics, 2016, no.6, pp. 9-14. doi: 10.20998/2074272X.2016.6.02.
5. Eyhab E.-K. Design and analysis of rolled rotor switched reluctance motor. Journal of Electrical Engineering \& Technology, 2006, Vol.1, No.4, pp. 472-481. doi: 10.5370/jeet.2006.1.4.472.

6. Bock U. A novel approach of modeling SR motor systems. ICEM 2002. 15th International Conference on Electrical Machines. Bruges, Belgium. 2002. p. 338. tromechanical converter with rolling rotor and axial air-gap. ACTA Electrotehnica, 2015, Vol.56, No.4, pp. 137-142.

8. Arkkio A., Biernat A., Bucki B., Kaminski G., Smak A., Staszewski P. Simulation model and laboratory test of switched reluctance motor with rolling rotor. Prace Naukowe Instytutu Maszyn, Napędów i Pomiarów Elektrycznych Politechniki Wroctawskiej, 2008, no.28, pp. 52-57.

9. Nanii V.V., Lykah V.A., Masliennikov A.M. Rotor motion in the rolling-rotor electrical machine. Transactions of Kremenno.3(86), pp. 9-13. (Rus).

10. Baguta V. A., Kulinchenko G. V. Evaluation of characteristics of mechatronic module based on a stepper motor. Bulletin of NTU «KhPI». Series: Problems of electrical machines and apparatus perfection. The theory and practice, 2013, no.51(1024),

\section{Received 10.09.2016}

G.V. Kulinchenko ${ }^{1}$, Candidate of Technical Science, Associate Professor,

A.M. Maslennikov ${ }^{2}$, Candidate of Technical Science, Associate Professor,

V.A. Baguta ${ }^{1}$, Engineer,

${ }^{1}$ Sumy State University,

2, Rymskogo-Korsakova Str., Sumy, 40007, Ukraine,

e-mail: heorhy@yandex.ua, viktrbaguta@gmail.com

${ }^{2}$ National Technical University «Kharkiv Polytechnic Institute», 21, Kyrpychova Str., Kharkiv, 61002, Ukraine,

e-mail: x-maslennikov@yandex.ua
7. Ungureanu C., Rata M., Graur A. PLC control of an elecchuk Mykhailo Ostrohradskyi National University, 2014, pp.43-53. (Rus). 